CERN-PH-TH/2007-155

\title{
Dark Energy, scalar-curvature couplings and a critical acceleration scale
}

\author{
Ignacio Navarro* \\ CERN, Theory Division, CH-1211 Geneva 23, Switzerland.
}

\begin{abstract}
We study the effects of coupling a cosmologically rolling scalar field to higher order curvature terms. We show that when the strong coupling scale of the theory is on the $10^{-3}-10^{-1} \mathrm{eV}$ range, the model passes all experimental bounds on the existence of fifth forces even if the field has a mass of the order of the Hubble scale in vacuum and non-suppressed couplings to SM fields. The reason is that the coupling to certain curvature invariant acts as an effective mass that grows in regions of large curvature. This prevents the field from rolling down its potential near sources and makes its effects on fifth-force search experiments performed in the laboratory to be observable only at the sub-mm scale. We obtain the static spherically symmetric solutions of the theory and show that a long-range force appears but it is turned on only below a fixed Newtonian acceleration scale of the order of the Hubble constant. We comment on the possibility of using this feature of the model to alleviate the CDM small scale crisis and on its possible relation to MOND.
\end{abstract}

\footnotetext{
${ }^{*}$ E-mail: ignacio.navarro@cern.ch
} 
If we want to obtain a scalar field model of dynamical Dark Energy (DE) its mass has to be of the order of the Hubble scale $1\left(H_{0} \sim 10^{-33} \mathrm{eV}\right)$ so that it evolves in the appropriate time-scale. This, of course, raises the question of how to avoid conflicts with the stringent limits on the (non) existence of extra long range forces coming from laboratory experiments and Solar System observations. Even if the scalar field couples to ordinary matter only with non-renormalizable interactions suppressed by the Planck scale, laboratory limits imply that its mass should be larger than $10^{-3} \mathrm{eV}$ [1]. Several mechanisms such as approximate global symmetries to suppress its couplings [2] or an environment-dependent effective potential and mass (the so-called chameleon mechanism) [3,4] have been proposed to reconcile a cosmologically rolling scalar field with the negative results of fifth-force search experiments. In this letter we consider the effect of coupling the quintessence field to higher order curvature invariants. We will see that such couplings provide a new way to suppress the effects of the force mediated by the scalar field at the Solar System or laboratory level and, at the same time, offer a range of unique observational signatures detectable in laboratory experiments or astrophysical observations. Specifically, the coupling we will be interested in is

$$
\Delta \mathcal{L}=\frac{\phi^{2}}{2 \Lambda^{2}} R_{\mu \nu \lambda \sigma} R^{\mu \nu \lambda \sigma}
$$

where $\phi$ is the ultra-light field, $R_{\nu \lambda \sigma}^{\mu}$ the Riemann curvature tensor and $\Lambda$ some mass scale. Its origin could be in a coupling of the scalar field with the Gauss-Bonnet combination, like in the DE models of [5], but since the scalar curvature or Ricci tensor part of such a coupling would not play any role in what follows we omit them for simplicity. And differently from the models in [5] we will not use this kind of coupling to drive the acceleration. We will assume that the field $\phi$ has an appropriate quintessence-like potential to drive the acceleration and this coupling can be simply interpreted as a curvature-dependent mass for the scalar field. Its effect will be to keep the field firmly locked at $\phi=0$ in regions of large curvature, e.g. near sources. However, on a cosmological scale the field will have a small mass at late times and will be able to roll down its potential. But for this to happen we must require that the field mass obtained from this operator is small enough today $\left(\Delta m_{s}^{2} \lesssim H_{0}^{2}\right)$. On a cosmological scale the operator above will translate into a mass of order

$$
\Delta m_{s}^{2} \sim \frac{H_{0}^{4}}{\Lambda^{2}}
$$

\footnotetext{
${ }^{1}$ We will use natural units throughout the paper so that $c$ and $\hbar$ are set to one.
} 
so the scale $\Lambda$ has to satisfy $\Lambda \gtrsim H_{0} \sim 10^{-33} \mathrm{eV}$ for the field to be able to roll cosmologically. On the other hand if we want the theory to pass the laboratory tests even if the field has non-suppressed couplings to matter we need its mass to be $m_{s} \gtrsim 10^{-3} \mathrm{eV}$ locally. In the neighborhood of a massive object like the Earth the Kretchmann scalar reads $R_{\mu \nu \lambda \sigma} R^{\mu \nu \lambda \sigma}=48 \frac{(G M)^{2}}{r^{6}}$, where $G$ is Newton's constant and $M$ is the mass of the object, so that near sources the field gets a mass

$$
m_{s}^{2} \simeq 48\left(\frac{G M}{r^{3} \Lambda}\right)^{2}
$$

Plugging in this expression the Earth's mass and radius and imposing that this mass is larger than $10^{-3} \mathrm{eV}$ we get the bound ${ }^{2} \Lambda \lesssim 10^{-31} \mathrm{eV}$. So we see that these constraints leave open just a small window for the scale $\Lambda$ of two orders of magnitude. And notice that this window is in an extremely small mass range of the order of the Hubble scale, $10^{-33}-10^{-31} \mathrm{eV}$, so one might wonder whether this theory could make sense as an effective quantum field theory at all. But to obtain the correct strong coupling scale of the theory we have to take into account the proper (canonical) normalization of the fluctuations of the metric. It is easy to see that the coupling (1), in a weak field expansion in flat space in powers of the fluctuations of the metric will give rise to couplings like

$$
\frac{\phi^{2}}{\Lambda^{2}} R_{\mu \nu \lambda \sigma} R^{\mu \nu \lambda \sigma}=\frac{\phi^{2} \partial^{2} h^{(c)} \partial^{2} h^{(c)}}{\Lambda^{2} M_{p}^{2}}+\mathcal{O}\left(\frac{h^{(c)}\left(\partial^{2} h^{(c)}\right)^{2}}{\Lambda^{2} M_{p}^{3}}\right)
$$

where we have suppressed the indices, and $h^{(c)}$ represents the canonically normalized metric fluctuations: $g_{\mu \nu}=\eta_{\mu \nu}+h_{\mu \nu}^{(c)} / M_{p}$. We see then that the strong coupling scale in this theory is $\Lambda_{s} \simeq\left(\Lambda M_{p}\right)^{1 / 2}$ that will be in the range $10^{-3}-10^{-1} \mathrm{eV}$. So our scalar field will be strongly coupled to gravity, with a strong coupling scale close to the vacuum energy scale 3 . And notice also that the mass of the field locally in the Earth is pushed close to, or might even get above, the cut-off scale of the theory. So, depending on the chosen parameters, we might get out of the range of validity of the theory when computing its implications for laboratory searches of fifth forces. But

\footnotetext{
${ }^{2}$ Over the distance scales associated with laboratory experiments we can take the field mass as constant. And as long as the density of our probe is less than the Earth's, the Earth's gravitational field will give the dominant contribution to the scalar mass.

${ }^{3}$ In fact it has been argued in [6] that taking a cut-off in our effective gravitational theory at the vacuum energy scale might be a way to understand the smallness of the cosmological constant (see however [7]).
} 
in any case we can expect that, like in the chameleon models of [3], the mass of the field will decrease and the range of the force mediated by this field will increase if an experiment is performed in space offering a very characteristic experimental signature.

But even if the field is massive close to sources it is nearly massless in regions of small curvature. It is therefore natural to ask what is the effect of the coupling (11) for long range forces. In order to gain some intuition about the expected behavior of the spherically symmetric solutions we can present the following argument. We expect that generically a massive boson will mediate a fifth force appreciable when the distance to the source is smaller than its inverse mass. In these situations the effect of the mass term in the equation of motion is negligible as compared with the terms coming from the Laplacian (i.e. momentum scale). We can approximate then the equation to solve by $\nabla^{2} \phi=\phi^{\prime \prime}+2 \phi^{\prime} / r=0$, yielding the usual $\phi \propto 1 / r$ profile characteristic massless fields. But for longer distances the mass term is not negligible anymore with respect to the derivative terms in the equations and this suppresses exponentially the solution. This well-known Yukawa exponential suppression $\left(\phi \propto e^{-m_{s} r} / r\right)$ is the reason why massive particles mediate only short range forces, significant only when $r<m_{s}^{-1}$. But now our effective mass term reads $\Delta m_{s}^{2} \sim 48\left(\frac{G M}{r^{3} \Lambda}\right)^{2}$ in the Schwarzschild geometry, so it depends on the distance to the source. And according to the previous argument, if the coupling above gives the dominant contribution to the mass we can expect an appreciable effect only whenever

$$
r<m_{s}(r)^{-1} \sim \frac{\Lambda r^{3}}{G M} .
$$

Since now the mass grows at short distance this condition means that the force would only appear at long distances, $r>r_{c}$, where $r_{c}$, defined as

$$
\frac{G M}{r_{c}^{2}} \equiv \Lambda,
$$

corresponds to a fixed gravitational acceleration $4 a_{c}=\Lambda$. We will confirm this result by explicitly obtaining the static spherically symmetric solutions to the equation of motion for the field $\phi$ with this $r$-dependent mass term. The fact that the force mediated by this field appears only below a fixed Newtonian acceleration scale makes this model

\footnotetext{
${ }^{4}$ This dependence of the mass with the distance yielding a fixed Newtonian acceleration as the threshold for the appearance of new effects is the same one finds for the scalar mode of the metric in the purely gravitational model of $[8]$.
} 
interesting from a phenomenological point of view, since it can produce the emergence of a critical acceleration in dark matter (DM) halos helping with the problems of conventional cold dark matter (CDM) models at small scales.

Let us present here a brief review of these problems, that will serve as an extra motivation for the kind of phenomenology that the present model can offer. The reader uninterested in this discussion can skip directly to the next paragraph. It can be said that the comparison of the structure of galaxies and DM halos formed in numerical simulations (within the $\Lambda \mathrm{CDM}$ paradigm) with their real observational counterparts is far from satisfactory. The main discrepancies come from the smallest scales: the simulations yield too much DM density in the central part of collapsed structures and too many sub-structures within DM halos (see e.g. [9,10] and references therein). This is the main cause of what is sometimes called the CDM "small scale crisis", and it has prompted the consideration of other flavors of DM, such as self-interacting [10], warm [11] or decaying DM [12]. However it is unclear if a DM self-interaction is compatible with the structure at other scales like cluster halos [13]. Also, the possibility of having warm DM is severely constrained by observations of Ly- $\alpha$ forest [14] and it does not seem compatible with the evolved structure seen at high redshifts, since it acts delaying structure-formation [15] (and the same constraints limit decaying DM scenarios [16]). But on top of this, there are certain systematic features of galactic dynamics that seem hard to explain in any conventional DM scenario. In particular the onset of the mass discrepancy or need for DM seems to be tightly correlated with a critical acceleration scale 5 , such that when the Newtonian acceleration produced by any given source is above it, there is no evidence for DM and when it is below we need to assume the presence of unseen forms of matter to explain the dynamics [17]. And this critical acceleration scale is very small, curiously of the order of the current Hubble constant. The above considerations, plus the cosmological constant problem, could be interpreted as hints of the existence of non-trivial physics within the "dark sector" 6 . And the mechanism presented here seems a good candidate to alter the physics of the infrared domain of gravity in the right direction. It can explain the appearance of a fixed acceleration scale in DM halos and has the potential to save some of the

\footnotetext{
${ }^{5}$ This empirical fact underlies the success of MOND, a phenomenological modification of Newton's potential for small accelerations that is able to predict with remarkable accuracy the rotation curve of spiral galaxies from its visible matter content [18.

${ }^{6}$ These motivations are of a theoretical nature for the DE, but purely observational for DM.
} 
contenders of CDM like warm or decaying DM by modifying the physics of structure formation because it can be seen as an enhancement of gravity in low density regions.

We shall continue now exploring the implications of this kind of interaction for long range forces. As usual we will assume a coupling of this field to ordinary matter such that in the non-relativistic limit it can be parametrized as

$$
\Delta S_{i n t}=\int d^{4} x \sqrt{g} \beta \frac{\phi}{M_{p}} \rho
$$

where $\rho$ is the matter energy density and $\beta$ a dimensionless parameter. The equation of motion for the scalar field reads then

$$
\square^{2} \phi+\frac{Q}{\Lambda^{2}} \phi=\beta \frac{\rho}{M_{p}}
$$

where we have defined $Q$ as the Kretchmann scalar $Q \equiv R_{\mu \nu \lambda \sigma} R^{\mu \nu \lambda \sigma}$. We are neglecting the potential for $\phi$ since we are assuming that we are in the regime where the coupling (11) gives the dominant contribution to the mass and the terms in this equation coming from the potential $V(\phi)$ are negligible. To find a solution one has to specify $Q(x)$, i.e the background geometry. In principle this should be done self-consistently, which means solving at the same time the coupled gravitational equations taking into account the back-reaction of the scalar field configuration in the background geometry. We will neglect now this backreaction and we will check later the conditions under which this is actually a good approximation. In this case the Kretchmann scalar outside a spherical mass distribution reads $Q=48\left(\frac{G M}{r^{3}}\right)^{2}$, so the equation that we have to solve in vacuum in order to find the static solutions is

$$
\frac{d^{2} \phi}{d r^{2}}+\frac{2}{r} \frac{d \phi}{d r}-48\left(\frac{G M}{\Lambda r^{3}}\right)^{2} \phi=0 .
$$

The general solution to this equation takes the form

$$
\phi(r)=\frac{\mathcal{C}_{1}}{\sqrt{r}} I_{-1 / 4}(z)+\frac{\mathcal{C}_{2}}{\sqrt{r}} K_{1 / 4}(z)
$$

where $I_{n}(z)$ and $K_{n}(z)$ are the modified Bessel functions of the first and second kind respectively. We have defined $z \equiv \frac{2 \sqrt{3} G M}{\Lambda r^{2}}$ and the $\mathcal{C}_{i}$ are the integration constants. Notice that $z(r)$ is basically the ratio of the gravitational acceleration to the critical acceleration $a_{c}=\Lambda$. To determine the integration constants we have to satisfy the appropriate boundary conditions at infinity and in the source. As we said the field will 
go to zero as we approach the source 7 . For large $z$ (or small $r$ ) we can approximate $I_{-1 / 4}(z) \propto e^{z} / z^{1 / 2}$ and $K_{1 / 4}(z) \propto e^{-z} / z^{1 / 2}$. So requiring that the solution is well behaved at small $r$ (remember that $z \propto 1 / r^{2}$ ) we see that we are forced to take $\mathcal{C}_{1}=0$. Notice that these solutions are analogous to the familiar $\phi \propto e^{ \pm m_{s} r} / r$ solutions for massive fields. Indeed, the heuristic argument presented before is backed by these exact solutions since $e^{-z} \sim e^{-m_{s}(r) r}$, and $\phi \propto I_{-1 / 4}(z) / \sqrt{r}$ is the analogous to the unphysical solution $\phi \propto e^{+m_{s} r} / r$ for massive fields. But curiously the role of the boundary conditions at the source and at infinity is reversed now with respect to the conventional case of a field with constant mass. In the conventional case we take the field to be asymptotically in its vacuum, say at $\phi=0$, so the coefficient of the $e^{+m_{s} r} / r$ solution is taken to be zero. The coefficient of the $e^{-m_{s} r} / r$ term is obtained by satisfying the boundary conditions at the source giving the source mass as the constant of proportionality (the solution would be $\phi=\beta \frac{M}{4 \pi M_{p}} \frac{e^{-m_{s} r}}{r}$ for a coupling to matter like the one in (7)). Now the field is locked at $\phi=0$ at short distances, forcing as we said $\mathcal{C}_{1}=0$, while we allow it to be rolling at infinity, where it will have an unspecified, time dependent v.e.v. that we will call $\phi_{\infty}$. As $r \rightarrow \infty$ we get the limit

$$
K_{1 / 4}(z) / \sqrt{r} \rightarrow 2 \cdot 3^{-1 / 8} \Gamma(5 / 4)\left(\frac{\Lambda}{G M}\right)^{1 / 4} \sim 1.58\left(\frac{\Lambda}{G M}\right)^{1 / 4},
$$

where $\Gamma(x)$ is the Euler gamma function. So the statid 8 solution taking into account this boundary condition at infinity will be

$$
\phi(r)=\frac{\phi_{\infty} 3^{1 / 8}}{2 \Gamma(5 / 4)}\left(\frac{G M}{\Lambda}\right)^{1 / 4} \frac{1}{\sqrt{r}} K_{1 / 4}(z) \simeq \frac{\phi_{\infty}}{1.58}\left(\frac{G M}{\Lambda}\right)^{1 / 4} \frac{1}{\sqrt{r}} K_{1 / 4}(z) .
$$

As we said this solution is exponentially suppressed at small distances (for $z>1$ ) but at large distances it asymptotes to $\phi_{\infty}$ with a $1 / r$ profile as can be seen from the large distance expansion

$$
\begin{aligned}
\phi(r) & \simeq \phi_{\infty}\left(1+\frac{3^{1 / 4} \Gamma(-1 / 4)}{\Gamma(1 / 4)}\left(\frac{G M}{\Lambda}\right)^{1 / 2} \frac{1}{r}+\mathcal{O}\left(\frac{G^{2} M^{2}}{\Lambda^{2} r^{4}}\right)\right) \\
& \simeq \phi_{\infty}\left(1-1.78\left(\frac{G M}{\Lambda}\right)^{1 / 2} \frac{1}{r}+\mathcal{O}\left(\frac{G^{2} M^{2}}{\Lambda^{2} r^{4}}\right)\right) .
\end{aligned}
$$

\footnotetext{
${ }^{7}$ Actually, inside the source the equilibrium value for the field will be displaced from zero because the source acts as a tadpole through the coupling (7), but the field will go to zero exponentially on a distance scale $\sim m_{s}^{-1}$ once we get out of the source.

${ }^{8}$ In reality the solution is not static because we are considering time-dependent asymptotic boundary conditions. But we are assuming here that the relaxation time of the system is much smaller than the time-scale associated with the change in boundary conditions $\left(\sim H_{0}^{-1}\right)$, so this static solution is a good approximation at any given time.
} 


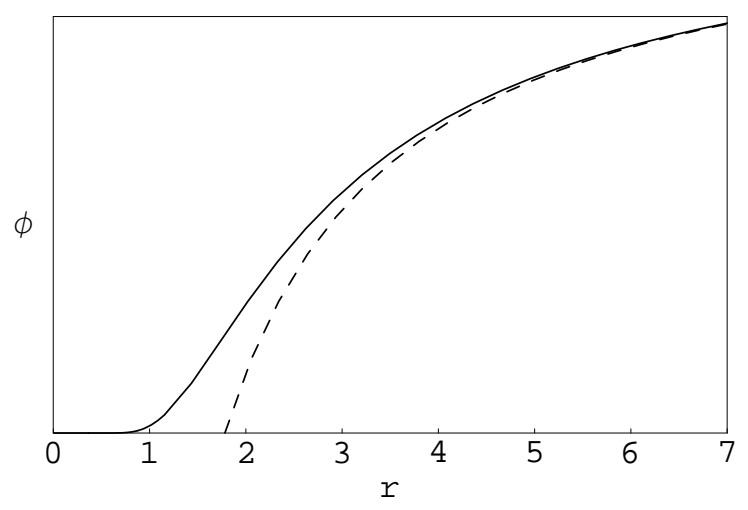

Figure 1: The solution for the scalar field with $r_{c}=1$. The dashed line corresponds to the asymptotic $1 / r$ dependence.

And notice that although this solution depends on the source mass and asymptotic field value, it is independent of the coupling of the field to matter, eq.(7). We also see how we recover the usual $1 / r^{2}$ falloff in the force caused by $\phi$ when $r \gg r_{c}$. In fig.1 we present a plot of this solution and in fig.2 a plot of its gradient, that would correspond to the force that a test particle would feel due to the profile of $\phi$ in this spherically symmetric solution. It is apparent how this force is switched on when $z \sim 1$ (or $r \sim r_{c}$ ) and gets exponentially suppressed at smaller distances.

But we still have to check that the backreaction of the scalar field configuration on the background is actually negligible, so that taking $Q=48\left(\frac{G M}{r^{3}}\right)^{2}$ is a good approximation. Since the scalar field is exponentially suppressed for $r<r_{c}$, we know that the backreaction is negligible in this regime and the solution is valid. We will check now under which conditions neglecting the backreaction for $r>r_{c}$ is justified. This can be quantified by looking at the $\phi$-dependent terms in the gravitational equations. The relevant terms in the equations of motion for the metric read

$$
\begin{aligned}
M_{p}^{2}\left(R_{\mu \nu}-\frac{1}{2} g_{\mu \nu} R\right) & =T_{\mu \nu}^{(\phi)}+\frac{2}{\Lambda^{2}} \nabla_{\sigma} \nabla_{\lambda}\left(R_{(\mu \nu}^{\sigma \lambda}{ }_{\nu} \phi^{2}\right) \\
& -\frac{\phi^{2}}{\Lambda^{2}} R_{\sigma \lambda \gamma \mu} R_{\nu}^{\sigma \lambda \gamma}+g_{\mu \nu} \frac{\phi^{2}}{4 \Lambda^{2}} R_{\sigma \lambda \gamma \kappa} R^{\sigma \lambda \gamma \kappa}
\end{aligned}
$$

where $T_{\mu \nu}^{(\phi)}$ is the usual energy-momentum tensor for $\phi$. We can now interpret the right hand side of these equations as an "effective energy-momentum density", $\mathcal{T}_{\mu \nu}^{(e f f)}$, that will have an influence on the geometry. So if the "energy-momentum" stored in this r.h.s. of the Einstein equations is small compared to $M$ we can neglect its influence on the background, while if it was bigger it can have a significant impact on 


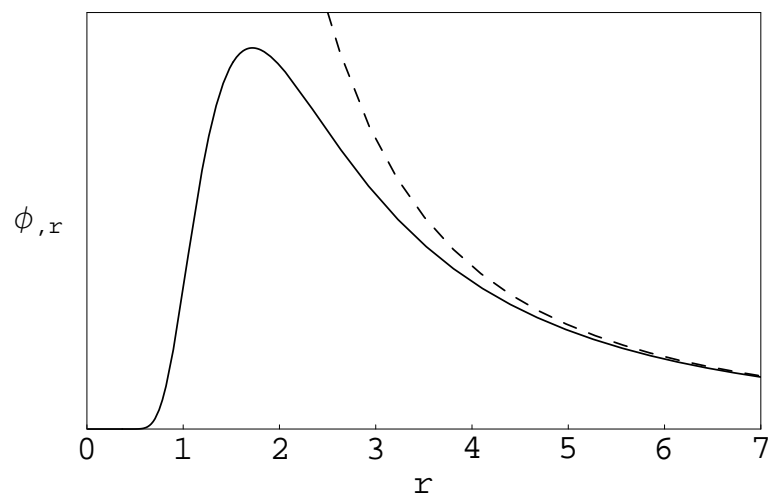

Figure 2: The gradient of the scalar field (i.e. the force) with $r_{c}=1$. The dashed line corresponds to the asymptotic $1 / r^{2}$ dependence.

the long-distance geometry. We are interested thus on the integration of this quantity as compared to $M$ at any given radius. As mentioned before it is obvious that for $r<r_{c}$ we can neglect $\mathcal{T}_{\mu \nu}^{(e f f)}$ because $\phi$ is exponentially suppressed. It is easy to check that asymptotically in our solution, when $r \gg r_{c}$ and $\phi \sim 1 / r$, the r.h.s. of the gravitational equations has the dependence

$$
\mathcal{T}_{\mu \nu}^{(e f f)} \sim \frac{\phi_{\infty}^{2} G^{2} M^{2}}{\Lambda^{3} r^{7}}
$$

In order to get an estimation of the order of magnitude of the integration of $\mathcal{T}_{\mu \nu}^{(e f f)}$ we will approximate $\phi$ by this long distance solution for $r>r_{c}$ and by zero for $r<r_{c}$. We get then

$$
M^{(e f f)}=\int d^{3} x \mathcal{T}_{00}^{(e f f)} \sim \int \frac{\phi_{\infty}^{2} G^{2} M^{2}}{\Lambda^{3} r^{7}} r^{2} d r \sim \frac{\phi_{\infty}^{2} G^{2} M^{2}}{\Lambda^{3} r_{c}^{4}} \sim \frac{\phi_{\infty}^{2}}{\Lambda} .
$$

Comparing this with $M$ we see that in order to be able to neglect the backreaction we must have $\phi_{\infty} \ll(\Lambda M)^{1 / 2}$.

It is also interesting to compare the usual Newtonian gravitational force with the long distance force that will appear when $r>r_{c}$ as a result of this profile for the scalar field. Their ratio, for $r \gg r_{c}$, is

$$
\frac{\beta \phi(r) / M_{p}}{G M / r} \sim \frac{\beta \phi_{\infty}}{(\Lambda M)^{1 / 2}}
$$

Notice that, when $\beta$ is of order one, the condition that we need for the backreaction of the scalar field on the geometry to be negligible is precisely that this ratio is small. So if $\beta \sim 1$ and the extra force due to the scalar becomes of the order of the gravitational 
one we can not neglect the influence of the scalar field configuration on the background geometry and our solution is not valid9. And since $(\Lambda M)^{1 / 2} \ll M_{p}$ for any reasonable $M$, this ratio will be very large unless $\phi_{\infty} \ll M_{p}$ as well, so we can only use the solutions we have presented when the v.e.v. of the field at infinity is small compared to the Planck mass. But although the precise form of the solution might be different when the backreaction is not negligible, we can still expect that the solution will be suppressed for $r<r_{c}$ (since the backreaction is negligible in this regime) and will recover a $\phi \propto 1 / r$ profile at large distances, $r \gg r_{c}$ (since the whole mass term for the scalar will be negligible in this regime and the field will behave as effectively massless). Nevertheless, it would be interesting to explore in more detail the behavior of the solutions when the backreaction is not negligible. In this case the solution for the metric will suffer an appreciable modification (with respect to the Schwarzschild solution), and this brings hopes that a model along these lines could be used to build MOND-like modification of gravity that bypasses the need for CDM. When the backreaction is negligible and the solution for the metric is not significantly altered we can not hope to build a realistic model that does not need DM because the effects of DM are also felt in gravitational lensing, and this effect can not be achieved without modifying the actual solution for the metric at long distances (see e.g. [19]).

To conclude, we have explored the effects of coupling the quintessence field to higher order curvature invariants and in particular we have focused in a coupling that can be interpreted as a curvature-dependent mass for the scalar field. On a cosmological scale the field mass is small so it can roll down its potential acting as dynamical Dark Energy. But as we approach sources this mass grows, driving the field towards zero, and we have found that if this coupling is strong enough it can shield the Solar System from long range forces mediated by this scalar. It is also worth mentioning that this effect also implies that no time variation of fundamental constants associated with this scalar field would be observed in local experiments 10. However, in the places where the field is rolling, basically inter-stellar or inter-galactic space, a time dependence of fundamental constants could be observed. So this kind of model has also the potential to reconcile the claims of the detection of a time evolution of fundamental constants

\footnotetext{
${ }^{9}$ However, if $\beta \gg 1$ we can still have an extra force stronger than gravity appearing at long distances while the backreaction is small, so we can trust our solution.

${ }^{10} \mathrm{Or}$ in the early Universe, since the field mass would be much bigger than the Hubble constant at early cosmological times and the field would be settled in its high-curvature minimum at $\phi=0$.
} 
derived from quasar absorption spectra with conflicting bounds obtained locally, like those coming from the natural Oklo reactor (see [20] for a review). Furthermore, the model also offers the opportunity to be tested in table-top laboratory experiments, and like the chameleon models of [3] boasts the characteristic signature of mediating a force whose range would be larger on space, where the local curvature is significantly lower. Needless to say, this theory has many more implications for astrophysics because at long distances an extra force will appear, becoming noticeable only when the gravitational acceleration produced by sources fall below a given scale. This feature makes the model interesting from a phenomenological point of view, since it appears to have the right properties to help with the problems of CDM at small scales. Interestingly, the strength of this force depends on the source's mass and asymptotic value of the field, but it is independent of the coupling of the field to matter. However a more detailed study of the effects of this kind of interaction in the formation and evolution of structures is beyond the scope of the present paper.

\section{Acknowledgments}

I would like to thank G. Efstathiou and K. Van Acoleyen for conversations.

\section{References}

[1] E. G. Adelberger, B. R. Heckel, S. Hoedl, C. D. Hoyle, D. J. Kapner and A. Upadhye, Phys. Rev. Lett. 98 (2007) 131104 arXiv:hep-ph/0611223]; D. J. Kapner, T. S. Cook, E. G. Adelberger, J. H. Gundlach, B. R. Heckel, C. D. Hoyle and H. E. Swanson, Phys. Rev. Lett. 98 (2007) 021101 arXiv:hep-ph/0611184.

[2] S. M. Carroll, Phys. Rev. Lett. 81 (1998) 3067 arXiv:astro-ph/9806099.

[3] J. Khoury and A. Weltman, Phys. Rev. Lett. 93 (2004) 171104 arXiv:astro-ph/0309300, $\quad$ Phys. Rev. D $69 \quad$ (2004) 044026 arXiv:astro-ph/0309411].

[4] N. Kaloper, arXiv:0706.1977 [astro-ph].

[5] S. Nojiri, S. D. Odintsov and M. Sasaki, Phys. Rev. D 71 (2005) 123509 arXiv:hep-th/0504052; L. Amendola, C. Charmousis and S. C. Davis, JCAP 0612 
(2006) 020 arXiv:hep-th/0506137, arXiv:0704.0175 [astro-ph]; T. Koivisto and D. F. Mota, Phys. Lett. B 644 (2007) 104 [arXiv:astro-ph/0606078], Phys. Rev. D 75 (2007) 023518 [arXiv:hep-th/0609155].

[6] S. R. Beane, Gen. Rel. Grav. 29 (1997) 945 [arXiv:hep-ph/9702419]; R. Sundrum, JHEP 9907 (1999) 001 arXiv:hep-ph/9708329, Phys. Rev. D 69 (2004) 044014 arXiv:hep-th/0306106.

[7] J. Polchinski, arXiv:hep-th/0603249.

[8] I. Navarro and K. Van Acoleyen, JCAP 0609 (2006) 006 arXiv:gr-qc/0512109, arXiv:astro-ph/0605322.

[9] J. P. Ostriker and P. J. Steinhardt, $\quad$ Science $300 \quad$ (2003) 1909 arXiv:astro-ph/0306402.

[10] D. N. Spergel and P. J. Steinhardt, Phys. Rev. Lett. 84 (2000) 3760 arXiv:astro-ph/9909386.

[11] P. Bode, J. P. Ostriker and N. Turok, Astrophys. J. 556 (2001) 93 arXiv:astro-ph/0010389.

[12] J. A. R. Cembranos, J. L. Feng, A. Rajaraman and F. Takayama, Phys. Rev. Lett. 95 (2005) 181301 arXiv:hep-ph/0507150; M. Kaplinghat, Phys. Rev. D 72 (2005) 063510 arXiv:astro-ph/0507300.

[13] N. Yoshida, V. Springel, S. D. M. White and G. Tormen, Astrophys. J. 535 (2000) L103 arXiv:astro-ph/0002362.

[14] U. Seljak, A. Makarov, P. McDonald and H. Trac, arXiv:astro-ph/0602430; M. Viel, J. Lesgourgues, M. G. Haehnelt, S. Matarrese and A. Riotto, Phys. Rev. D 71 (2005) 063534 arXiv:astro-ph/0501562, Phys. Rev. Lett. 97 (2006) 071301 arXiv:astro-ph/0605706.

[15] Z. Haiman, R. Barkana and J. P. Ostriker, arXiv:astro-ph/0103050.

[16] K. Jedamzik, M. Lemoine and G. Moultaka, JCAP 0607 (2006) 010 arXiv:astro-ph/0508141. 
[17] S. S. McGaugh, Astrophys. J. 609 (2004) 652 [arXiv:astro-ph/0403610].

[18] R. H. Sanders and S. S. McGaugh, Ann. Rev. Astron. Astrophys. 40 (2002) 263 arXiv:astro-ph/0204521.

[19] J. D. Bekenstein, Phys. Rev. D 70 (2004) 083509 [Erratum-ibid. D 71 (2005) 069901] arXiv:astro-ph/0403694].

[20] J. P. Uzan, Rev. Mod. Phys. 75 (2003) 403 arXiv:hep-ph/0205340. 